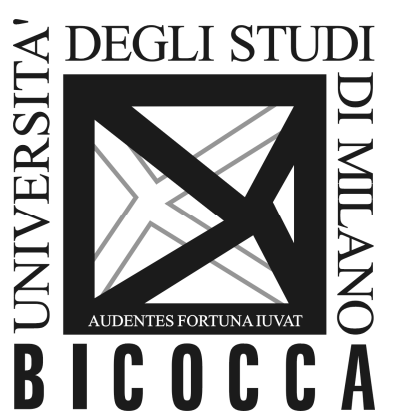

DEPARTMENT OF ECONOMICS,
MANAGEMENT AND STATISTICS
UNIVERSITY OF MILAN - BICOCCA

DEMS WORKING PAPER SERIES

Determinacy analysis in high order dynamic systems: The case of nominal rigidities and limited asset market participation

Guido Ascari, Andrea Colciago, Lorenza Rossi

No. 334 - April 2016

Dipartimento di Economia, Metodi Quantitativi e Strategie di Impresa

Università degli Studi di Milano - Bicocca

http://dems.unimib.it/ 


\title{
Determinacy analysis in high order dynamic systems: The case of nominal rigidities and limited asset market participation*
}

\author{
Guido Ascari $^{\dagger} \quad$ Andrea Colciago ${ }^{\ddagger} \quad$ Lorenza Rossi $^{\S}$
}

\begin{abstract}
We show how to use Hurwitz polynomials to study the stability and uniqueness of Rational Expectation equilibria in Dynamic General Equilibrium models. We apply this method to a model characterized by staggered wage and price contracts and by limited asset market participation (LAMP). We prove analytically in a fourth-order dynamics system that, once nominal wage stickiness is taken into account, LAMP does not invalidate the Taylor Principle: for any plausible degree of asset market participation an active interest rate rule ensures the uniqueness of the rational expectation equilibrium.
\end{abstract}

JEL Classification Numbers: C62, E50. hold.

Keywords: determinacy, high-order dynamics, sticky wages, non-Ricardian house-

*This note previously circulated as part of working paper titled "Limited Asset Market Participation: does it Really Matter for Monetary Policy?" (Bank of Finland WP 15-12). Andrea Colciago is grateful to the Central Bank of Finland for hospitality as a Visiting Schoolar while this paper was written. The authors thank the MIUR for financial support through a PRIN grant.

${ }^{\dagger}$ Afiliation: University of Oxford and University of Pavia. Email: guido.ascari@economics.ox.ac.uk.

${ }^{\ddagger}$ Afiliation: Department of Economics, University of Milano Bicocca and Research Department, Dutch National Bank. Email: andrea.colciago@gmail.com

$\S$ Affiliation: University of Pavia. Email: lorenza.rossi@eco.unipv.it. 


\section{Introduction}

The contribution of this paper is twofold. First, we show how to use Hurwitz polynomials to study the stability and uniqueness of Rational Expectation Equilibria (REE) in Dynamic General Equilibrium models. Hurwitz polynomials are used to transform the polynomial derived from the characteristic equation, as in Felippa and Park (2004), to deal analytically with the conditions for determinacy. ${ }^{1}$

Second, we apply our methodology to a New Keynesian (NK) model characterized by staggered wage and price contracts together with limited asset market participation (LAMP). We thus assess analytically how nominal wage stickiness affects the results in Bilbiie (2008). In our model wage and price stickiness arises from the standard Calvo-type mechanism. As in Galì et al. (2004, 2007), Bilbiie (2008) and in a number of recent studies, LAMP is modeled assuming that a portion of agents face a liquidity constraint such that they spend their current labor income in each period. The resulting framework nests two popular environments in the monetary policy literature: Bilbiie (2008) and Erceg et al. (2000). Bilbiie (2008) studies determinacy properties of simple interest rate rules and optimal monetary policy in a NK economy with LAMP and a frictionless labor market. In such a setting, determinacy of the REE requires the inversion of the Taylor principle, that is, the nominal interest rate needs to react less than one-to-one to inflation. Erceg et al. (2000) develop a full participation NK model characterized by both staggered prices and wages which features an endogenous trade-off between the stabilization of the output gap, price inflation and wage inflation.

Our model can be represented as a fourth order dynamic system characterized by both predetermined and forward looking variables. Despite the high order of the model dynamic system, our methodology allows us to provide analytical conditions for the determinacy of the REE. We prove that once nominal wage stickiness, an incontrovertible empirical fact, is taken into account, LAMP does not invalidate the Taylor Principle: for any plausible share of non-Ricardian agents an active interest rate rule ensures the uniqueness of the rational expectation equilibrium.

Colciago (2011) shows numerically that wage stickiness helps restoring the standard Taylor Principle as a necessary condition for determinacy in the presence of LAMP. We apply our methodology to prove analytically the generality of this numerical result. This is possible since our methodology allows to obtain analytical determinacy conditions in high order dynamic systems. As such, the proposed methodology will be a very useful instrument for many researchers in macroeconomics.

The remainder of the paper is organized as follows. Section 2 shows how to use Hurwitz polynomials for the study of uniqueness and stability, Section 3 applies the method to a NK model with LAMP and Section 4 concludes.

\section{Methodology}

Consider a system of linear difference equations in the form

$$
E_{t} z_{t+1}=A z_{t}
$$

where $z_{t}$ is a $n x 1$ vector including $n_{1}$ predetermined variables and $n_{2}$ non-predetermined variables, where $n=n_{1}+n_{2}$. A is a $n x n$ coefficient matrix. The characteristic polynomial

\footnotetext{
${ }^{1}$ In the remainder we use the terms uniqueness and determinacy as sinonimous.
} 
associated to matrix $\mathrm{A}$ is

$$
P_{C}(\gamma)=\gamma^{n}+a_{1} \gamma^{n-1}+. . a_{i} \gamma^{n-i}+. .+a_{n-1} \gamma+a_{n}
$$

The stability and uniqueness properties of the solution to (1) would depend on the location of the roots of $P_{C}(\gamma)$ inside the unit circle $|\gamma|<1$. In particular, Blanchard and Kahn (BK henceforth, 1980) show that (1) has a stable and unique solution if (2) has $n_{2}$ roots larger than one in absolute value and $n_{1}$ roots lower than one in absolute value. Verifying if BK conditions are satisfied can be cumbersome, particularly so as $n$ gets larger and when both $n_{1}$ and $n_{2}$ differ from zero, i.e. if $z_{t}$ contains both predetermined and non-predetermined variables.

To help obtaining analytical results on the stability properties of the dynamic system (2), Felippa and Park (2004) illustrates the strategy of transforming the polynomial $P_{C}(\gamma)$ in an Hurwitz polynomial, $P_{H}(s)$, by applying the conformal involuntary transformation

$$
\gamma=\frac{1+s}{1-s}
$$

Given (3), it is easy to check that $|\gamma| \lessgtr 1 \Leftrightarrow s \lessgtr 0$. Expanding the polynomial, one obtains a quotient of two polynomials: $\tilde{P}_{H}(s)=\frac{P_{H}(s)}{Q_{H}(s)}$ where the roots of $\tilde{P}_{H}(s)$ are the roots of $P_{H}(s)$. The uniqueness and stability properties of $P_{H}(s)$ depend on the location of the roots in the left-hand plane $\Re(s) \leq 0$. The dynamic system (1) has a stable and unique solution if the Hurwitz polynomial associated to (2) has $n_{2}$ roots larger than zero and $n_{1}$ roots lower than zero. To check how many roots are positive and how many are negative in a high order polynomial is a much simpler task than to check how many roots are within or outside the unit circle. Moreover, in standard microfounded macro-models the sign of the parameters defining functional forms is usually known, while their magnitude is not. As a result verifying if these conditions are satisfied is more straightforward then verifying if the traditional BK conditions are.

In the next section we apply the methodology just explained to a NK model with price and wage stickiness and LAMP. Importantly, despite the dynamic system is 4th order, we provide analytical determinacy conditions.

\section{Application: A NK model with nominal rigidities and LAMP}

To guide the reader through the methodology described above we consider a NK model characterized by: (i) staggered wage and price contracts; (ii) LAMP, that is, a fraction $\lambda \in$ $[0,1]$ of agents do not participate to the financial markets and simply consume their labor income. The model economy is spelled out in detail in Ascari et al. (2015). ${ }^{2}$ The following

\footnotetext{
${ }^{2}$ The reduce form of the model is derived under the assumption that each union pools the labor income of agents, leading Ricardian and non-Ricardian households to work for the same amount of time. This implies that under flexible wages the model does not fully nest Bilbiie (2008), where Ricardian and non-Ricardian agents are free to make different labor choices. However, our findings do not depend on the chosen structure of the labor market. In an on-line Appendix, available on the web-page of the authors, we investigate an alternative labor market arrangement that allows to fully nest Bilbiie (2008). This alternative labor market setting does not invalidate our results, which, on the contrary, are strengthened. We take the labor market setting in the main text as the baseline because we regard it as a more rigorously microfounded.
} 
equations summarize log-linear equilibrium dynamics of the model around the efficient steady state:
$(M 1) \quad \pi_{t}=\beta E_{t} \pi_{t+1}+\kappa_{p} \tilde{\omega}_{t}$
NKPC
(M2) $\pi_{t}^{w}=\beta E_{t} \pi_{t+1}^{w}+\kappa_{w}\left[(\sigma+\phi) x_{t}-\tilde{\omega}_{t}\right]$
Wage Inflation Curve
(M3) $\tilde{\omega}_{t}=\tilde{\omega}_{t-1}+\pi_{t}^{w}-\pi_{t}-\Delta \omega_{t}^{E f f}$
Real Wage Gap
(M4) $x_{t}=E_{t} x_{t+1}-\frac{1}{\sigma} E_{t}\left(i_{t}-\pi_{t+1}-r_{t}^{E f f}\right)-\frac{\lambda}{(1-\lambda)} E_{t} \Delta \tilde{\omega}_{t+1} \quad$ IS curve

We assume that fluctuations are determined by exogenous shocks to labor productivity and by taste shocks. The log-labor productivity is denoted by $a_{t}$, while taste shocks are denoted in $\operatorname{logs}$ by $\psi_{t}$. Equation $(M 1)$ is the NKPC obtained from the firms' price setting problem. The variable $\pi_{t}$ represent deviations of current inflation from its (zero) steady state, $\tilde{\omega}_{t}=\omega_{t}-\omega_{t}^{E f f}$ represents the real wage gap, which is defined as the gap between the current and the efficient equilibrium real wage. The latter is determined uniquely by technology, $\omega_{t}^{E f f}=a_{t}$. The parameter $\kappa_{p}=\frac{\left(1-\beta \xi_{p}\right)\left(1-\xi_{p}\right)}{\xi_{p}}$ is the slope of the NKPC, where $\beta$ is the subjective discount factor in the agents' utility function and $\xi_{p}$ is the Calvo-probability for a firm of not being able to change its price. The variable $\pi_{t}^{w}$ represents wage inflation and equation (M2) is a wage inflation curve, with slope $\kappa_{w}=\frac{\left(1-\beta \xi_{w}\right)\left(1-\xi_{w}\right)}{\xi_{w}}$, where $\xi_{w}$ is the Calvo-probability for a labor union of not being able to change its wage. The variable $x_{t}=y_{t}-y_{t}^{E f f}$ denotes the output gap, i.e. the gap between actual output and the efficient output, which reads as $y_{t}^{E f f}=$ $\frac{1+\phi}{\sigma+\phi} a_{t}+\frac{1}{(\sigma+\phi)} \psi_{t}$. The parameters $\phi$ and $\sigma$ are the elasticity of intertemporal substitution in labor supply and in consumption, respectively. Equation (M3) simply provides the definition of the real wage gap in terms of wage and price inflation and $\Delta \omega_{t}^{E f f}=\omega_{t}^{E f f}-\omega_{t-1}^{E f f}$. Equation (M4) represents the IS curve of this economy. The latter differs from a standard IS equation because of the extra term $\frac{\lambda}{1-\lambda} E_{t} \Delta \tilde{\omega}_{t+1}$. The expected growth of the real wage gap appears in the IS curve because it affects aggregate demand relative to the efficient allocation through the consumption of constrained consumers - it will not appear if $\lambda=0$. The efficient real rate of interest is defined as $r_{t}^{E f f}=\sigma\left(\frac{1+\phi}{\sigma+\phi} \Delta a_{t+1}-\frac{\phi}{\sigma(\sigma+\phi)} \Delta \psi_{t+1}\right)$. To close the model we consider the same interest rate rule as in Bilbiie (2008)

$$
i_{t}=\phi_{\pi} \pi_{t+1}
$$

Abstracting from shocks, the relevant system to study the determinacy of REE can be represented in matrix forms as in (1) with $z_{t}=\left[\pi_{t}^{w}, \pi_{t}, x_{t},, \tilde{\omega}_{t}\right]^{\prime}$ and

$$
A=\left[\begin{array}{cccc}
\frac{1}{\beta} & 0 & -\frac{1}{\beta} \kappa_{w}(\sigma+\phi) & -\frac{1}{\beta} \kappa_{w} \\
0 & \frac{1}{\beta} & 0 & -\frac{1}{\beta} \kappa_{p} \\
\frac{1}{\beta} \chi & \frac{1}{\sigma} \frac{1}{\beta}\left(\phi_{\pi}-1\right)-\chi \frac{1}{\beta} & 1-\chi \frac{1}{\beta} \kappa_{w}(\sigma+\phi) & \chi \frac{1}{\beta}\left(\kappa_{w}+\kappa_{p}\right)-\frac{1}{\sigma} \frac{1}{\beta}\left(\phi_{\pi}-1\right) \kappa_{p} \\
\frac{1}{\beta} & -\frac{1}{\beta} & -\frac{1}{\beta} \kappa_{w}(\sigma+\phi) & 1+\frac{1}{\beta}\left(\kappa_{w}+\kappa_{p}\right)
\end{array}\right]
$$

The 4th-order characteristic polynomial reads as

$$
P_{C}(\gamma)=\gamma^{4}+a_{1} \gamma^{3}+a_{2} \gamma^{2}+a_{3} \gamma+a_{4}
$$

The latter can be transformed into the Hurwitz polynomial by using $\gamma=\frac{1+s}{1-s}$. In this case

$$
\tilde{P}_{H}(s)=\left(\frac{1+s}{1-s}\right)^{4}+a_{1}\left(\frac{1+s}{1-s}\right)^{3}+a_{2}\left(\frac{1+s}{1-s}\right)^{2}+a_{3} \frac{1+s}{1-s}+a_{4} .
$$


Hence one needs to study the stability properties of the following Hurwitz polynomial

$$
P_{H}(s)=\underbrace{\tilde{a}_{4}}_{\frac{a_{1}+a_{2}+a_{3}+a_{4}+1}{a_{2}-a_{1}-a_{3}+a_{4}+1}}+s \underbrace{\tilde{a}_{3}}_{\frac{2\left(2+a_{1}-a_{3}-2 a_{4}\right)}{a_{2}-a_{1}-a_{3}+a_{4}+1}}+s^{2} \underbrace{\tilde{a}_{2}}_{\frac{2\left(3 a_{4}-a_{2}+3\right)}{a_{2}-a_{1}-a_{3}+a_{4}+1}}+s^{3} \underbrace{\tilde{a}_{1}}_{\frac{2\left(a_{3}-a_{1}-2 a_{4}+2\right)}{a_{2}-a_{1}-a_{3}+a_{4}+1}}+s^{4}
$$

Proposition 1. Determinacy conditions. Let $i_{t}=\phi_{\pi} \pi_{t+1}$. The REE is determinate iff:

1) either $\phi_{\pi} \in\left(1 ; \bar{\phi}_{\pi}^{F R}\right)$ if $\bar{\phi}_{\pi}^{F R}>1$;

2) or $\phi_{\pi} \in\left(\bar{\phi}_{\pi}^{F R} ; 1\right)$ if $\bar{\phi}_{\pi}^{F R}<1$;

where $\bar{\phi}_{\pi}^{F R}=1+\frac{2 \sigma(1+\beta)\left[2(1+\beta)+\kappa_{p}+\kappa_{w}-\frac{\lambda}{1-\lambda} \kappa_{w}(\sigma+\phi)\right]}{\kappa_{w} \kappa_{p}(\sigma+\phi)}$.

While the proof is in Appendix A.1, here we want to sketch it to show how to apply the methodology we propose. The key point is to apply the transformation in (6) to go from the characteristic polynomial of matrix $A$

$$
\begin{aligned}
P_{C}(\gamma)= & \gamma^{4}+\left[\frac{1}{\beta}\left[-2-2 \beta-\left(\kappa_{w}+\kappa_{p}\right)+\chi \kappa_{w}(\sigma+\phi)\right]\right] \gamma^{3} \\
& +\left[\frac{1}{\beta}\left(\kappa_{p}+\kappa_{w}+\beta+1\right)-\frac{1}{\beta} \kappa_{w}(\sigma+\phi)\left(\chi\left(1+\frac{1}{\beta}\right)+\frac{1}{\sigma \beta} \kappa_{p}\left(\phi_{\pi}-1\right)\right)\right. \\
& \left.+\frac{1}{\beta^{2}}\left(1+3 \beta+\kappa_{w}+\kappa_{p}\right)\right] \gamma^{2} \\
& +\left[-\frac{1}{\beta^{2}}\left(2+2 \beta+\kappa_{w}+\kappa_{p}-\chi \kappa_{w}(\sigma+\phi)\right)\right] \gamma+\frac{1}{\beta^{2}}
\end{aligned}
$$

to the associated Hurwitz polynomial

$$
\begin{aligned}
& P_{H}(s)=\underbrace{\frac{-\frac{1}{\sigma} \kappa_{w} \kappa_{p}(\sigma+\phi)\left(\phi_{\pi}-1\right)}{D}}_{\tilde{a}_{3}} \\
& +s \underbrace{\frac{2(\beta-1)\left[-\kappa_{w}-\kappa_{p}+\chi \kappa_{w}(\sigma+\phi)\right]}{D}}_{\tilde{a}_{4}} \\
& +s^{2} \underbrace{\left.\frac{4 \beta^{2}+4-8 \beta+2(1+\beta)\left[-\kappa_{p}-\kappa_{w}+\kappa_{w}(\sigma+\phi) \chi\right]+\frac{2}{\sigma} \kappa_{w}(\sigma+\phi) \kappa_{p}\left(\phi_{\pi}-1\right)}{D}\right]}_{\tilde{a}_{1}}] \\
& +s^{3}(\underbrace{\frac{-8+8 \beta^{2}+2(1-\beta)\left[-\left(\kappa_{w}+\kappa_{p}\right)+\chi \kappa_{w}(\sigma+\phi)\right]}{D}})+s^{4},
\end{aligned}
$$

where

$$
D=4 \beta^{2}+4+8 \beta+2[\beta+1]\left(\kappa_{p}+\kappa_{w}\right)-\frac{1}{\sigma} \kappa_{w} \kappa_{p}(\sigma+\phi)\left(\phi_{\pi}-1\right)-2(1+\beta) \chi \kappa_{w}(\sigma+\phi) .
$$

This polynomial should exhibit 3 positive roots and 1 negative root for the REE to be unique. This is a much easier condition to check than checking whether 3 roots and 1 root of $P_{C}(\gamma)$ 
are outside and inside the unit circle, respectively. The proof in the Appendix simply analyses the signs of the coefficients $\tilde{a}_{i}$, and exploits the Decartes' rule of sign for polynomials.

On top of the methodological aspect, Proposition 1 analytically illustrates the second contribution of the paper. As in Bilbiie (2008), there is a region of the parameter space where the Taylor principle is inverted: when $\bar{\phi}_{\pi}^{F R}<1, \phi_{\pi}$ needs to be lower than 1 to yield a unique REE. However, the condition $\bar{\phi}_{\pi}^{F R}<1$ implicitly defines a threshold value, $\bar{\lambda}^{F R}$, for the fraction of non-asset holders

$$
\bar{\phi}_{\pi}^{F R} \lessgtr 1 \Leftrightarrow \bar{\lambda}^{F R} \gtrless \frac{2(1+\beta)+\kappa_{w}+\kappa_{p}}{\kappa_{w}(\sigma+\phi)+2(1+\beta)+\kappa_{w}+\kappa_{p}} .
$$

The value of $\bar{\lambda}^{F R}$ depends on the model parameters, and, in particular, on the degree of wage stickiness.

Figure 1 depicts determinacy areas in the space $\left(\lambda, \phi_{\pi}\right)$. The solid curved line represents the threshold value $\bar{\phi}_{\pi}^{F R}$ described in Proposition 1 as a function of $\lambda$. Note that $\bar{\phi}_{\pi}^{F R}$ decreases with the degree of LAMP, $\lambda$. If $\lambda=0, \bar{\phi}_{\pi}^{F R}>1$, and the standard Taylor principle holds. As $\lambda$ increases, however, $\bar{\phi}_{\pi}^{F R}$ decreases, and the interval for $\phi_{\pi}$ described in case 1) of Proposition 3 shrinks and eventually becomes empty when $\lambda=\bar{\lambda}^{F R}$. As $\lambda$ increases further, then, condition 2) applies and the interval for $\phi_{\pi}$ in the inverted Taylor principle case enlarges, becoming $\phi_{\pi} \in(-\infty ; 1)$ at the limit when $\lambda \rightarrow 1$.

What is the effect of wage stickiness? Wage stickiness shifts to the right the $\bar{\phi}_{\pi}^{F R}$ curve, because $\frac{\partial \bar{\phi}_{\pi}^{F R}}{\partial \kappa_{w}}<0 .{ }^{3}$ Hence, the threshold value $\bar{\lambda}^{F R}$ increases with the degree of wage stickiness. As $\kappa_{w}$ tends to 0 , i.e. with fix wages, then $\bar{\phi}_{\pi}^{F R} \longrightarrow \infty$, and the Taylor principle is restored, because Proposition 1 guarantees determinacy if and only if $\phi_{\pi} \in(1 ; \infty)$. On the contrary, in the case of flexible wages $\left(\kappa_{w} \longrightarrow \infty\right)$ as in Bilbiie (2008), the threshold value becomes $\bar{\lambda}^{F R, f w}=\frac{1}{\sigma+\phi+1}$, that is lower than $\bar{\lambda}^{F R}$.

To give a quantitative flavour of Proposition 1, Figure 2 depicts indeterminacy regions in the parameter space $\left(\phi_{\pi}, \lambda\right)$, obtained by numerical simulations. ${ }^{4}$ Panel (i) displays the case of flexible wages. A share of non-Ricardian agents larger than 0.167 requires the inverted Taylor Principle to ensure equilibrium uniqueness. Thus, "the inverted Taylor principle holds 'generically' (i.e., if we exclude some extreme values for some of the parameters)"(Bilbiie, 2008 , p. 180). Panel (ii) refers to the case of sticky wages, with an average duration of wage contracts equal to three quarters. ${ }^{5}$ Unless the share of non-Ricardian consumers assumes rather extreme values - not compatible with existing estimates - the Taylor Principle leads to equilibrium determinacy. Thus, wage stickiness "generically" restores standard determinacy conditions.

\footnotetext{
${ }^{3}$ Recall that the higher the degree of stickiness in wages, the lower is $\kappa_{w}$.

${ }^{4}$ Our calibration is standard. Time is measured in quarters. The discount factor $\beta$ is set to 0.99 , so that the annual interest rate amounts to 4\%. The utility parameters $\sigma$ and $\phi$ are equal to 2 and 3, respectively. According to the estimates in Basu and Fernald (1997) the value added mark-up of prices over marginal cost is around $20 \%$, for this reason we set $\theta_{p}$ to 6 . We assign an identical value to the elasticity of substitution between labor inputs, $\theta_{w}$. We set $\xi_{p}=\xi_{w}=0.75$, which implies an average duration of price and wage contracts of one year, a value which is in compatible with most available empirical estimates (see for example Smets and Wouters 2003 and Levin et al. 2005). The Figure reproduces the main result in Colciago (2011).

${ }^{5}$ To understand that Figure 1 and panel (ii) of Figure 2 are equivalent, recall that Proposition 1 only focuses on the necessary and sufficient conditions for determinacy of the REE, and do not consider the difference between indeterminacy and instability whenever the REE is not unique. Moreover, given our calibration, the curve that defines $\bar{\phi}_{\pi}^{F R}$ in the space $\left(\phi_{\pi}, \lambda\right)$ is almost horizontal at $\bar{\lambda}^{F R}$ and it bends only for extreme values of $\lambda\left(\right.$ or $\left.\phi_{\pi}\right)$.
} 
Corollary. Numerical results. Let $i_{t}=\phi_{\pi} \pi_{t+1}$. Under sticky wages and sticky prices the Taylor Principle is a necessary condition for equilibrium determinacy for all the plausible parameterizations of the share of non-Ricardian agents.

To gain some intuition about how the interaction between non Ricardian agents and wage stickiness affects determinacy, consider the following mental experiment, which builds on Bilbiie (2008). After monetary policy increases in the interest rate, Ricardian agents reduce their demand, while the firms that cannot change their price reduce labor demand. The labor demand curve thus shifts inward and under flexible wages this translates into a reduction in the real wage - the more so the higher the elasticity of the marginal disutility of hours, $\phi$, and the inverse intertemporal elasticity of substitution in consumption, $\sigma$. The decrease in the real wage depresses demand by non-Ricardian agents and reinforces the effects on aggregate demand due to the initial increase in the real interest rate. However, as emphasized by Bilbiie (2008), this effect is not monotonic in $\lambda$. The sizeable decrease in the real wage, and hence in marginal costs, together with the small change in hours, and hence in output and sales, imply a potential increase in profits. This leads, in turn, to a positive wealth effect on Ricardian agents. The latter is stronger the larger $\lambda$, since Ricardian agents would obtain a higher individual dividend income. If asset market participation is restricted enough, the positive income effect may counteract the substitution effect induced by the interest rate change and finally lead to an increase in aggregate demand. Bilbiie (2008) names this region of the parameter space Inverted Aggregate Demand Logic, because it implies the inversion of the slope of the IS curve: an increase in the interest rate leads to an increase in aggregate demand. When this happens, then the Taylor principle obviously inverts too. Consider now the case of sticky wages. The inward shift in labor demand due to the reduction in consumption by Ricardian agents after the interest rate increase, now results in a modest reduction in the real wage because of wage stickiness. The increase in profits is thus dampened with respect to the case of flexible wages. For this reason under wage stickiness the inversion of the IS curve requires a much larger share of non Ricardian agents to magnify the, eventual, wealth effect at the individual level for the Ricardian agents (see Ascari et al., 2015). Hence, for any given share of non Ricardian agents, the inversion of the slope of the IS curve becomes more likely as wages become more flexible.

Finally, price stickiness has instead the opposite effect on $\bar{\lambda}^{F R}$ : the threshold value, $\bar{\lambda}^{F R}$ decreases with the degree of price stickiness (lower $\kappa_{p}$ ) because $\frac{\partial \bar{\lambda}^{F R}}{\partial \kappa_{p}}>0$. In the case of flexible prices and sticky wages, the model becomes isomorphic to a fully Ricardian economy, $\lambda$ would not matter and hence the standard Taylor principle applies: the REE is unique iff $\phi_{\pi} \in\left(1,1+\frac{2 \sigma(1+\beta)}{\kappa_{w}(\sigma+\phi)}\right)$.

\subsection{Contemporaneous Taylor rules}

First, we consider the contemporaneous rule $i_{t}=\phi_{\pi} \pi_{t}$. The Appendix shows that the method can be applied also in this case despite the high order dimension of the relevant matrix.

Proposition 2. Current price inflation targeting rule. Let $i_{t}=\phi_{\pi} \pi_{t}$. The REE is determinate iff:

1) either $\phi_{\pi}>\max \left\{1 ; \bar{\phi}_{\pi}^{a, C R} ; \bar{\phi}_{\pi}^{b, C R}\right\}$;

2) or $\phi_{\pi}<\min \left\{1 ; \bar{\phi}_{\pi}^{a, C R} ; \bar{\phi}_{\pi}^{b, C R}\right\}$; 
where $\bar{\phi}_{\pi}^{a, C R}=-1-\frac{2 \sigma(1+\beta)\left[2(1+\beta)+\left(\kappa_{p}+\kappa_{w}\right)-\frac{\lambda}{1-\lambda}(\sigma+\phi) \kappa_{w}\right]}{(\sigma+\phi) \kappa_{p} \kappa_{w}}$ and $\bar{\phi}_{\pi}^{b, C R}=\frac{\sigma(1-\beta)\left[\frac{\lambda}{1-\lambda}(\sigma+\phi) \kappa_{w}-\left(\kappa_{p}+\kappa_{w}\right)\right]}{(\sigma+\phi) \kappa_{p} \kappa_{w}}$.

Proof. See Appendix A.2.1. ${ }^{6}$

This case is different from the previous one. Figure 3 visualizes the determinacy regions in the $\left(\phi_{\pi}, \lambda\right)$ space. Note that the two curves defining $\bar{\phi}_{\pi}^{a, C R}$ and $\bar{\phi}_{\pi}^{b, C R}$ are now both increasing in $\lambda$. The two cases 1) and 2) in proposition 2 characterize two frontiers: $\max \left\{1 ; \bar{\phi}_{\pi}^{a, C R} ; \bar{\phi}_{\pi}^{b, C R}\right\}$ and $\min \left\{1 ; \bar{\phi}_{\pi}^{a, C R} ; \bar{\phi}_{\pi}^{b, C R}\right\}$, respectively. Determinacy, thus, occurs below the lower frontier $\left(\max \left\{1 ; \bar{\phi}_{\pi}^{a, C R} ; \bar{\phi}_{\pi}^{b, C R}\right\}\right)$ and above the upper frontier $\left(\min \left\{1 ; \bar{\phi}_{\pi}^{a, C R} ; \bar{\phi}_{\pi}^{b, C R}\right\}\right)$. In this case, it is impossible to define an "inversion of the Taylor principle". On the one hand, for each value of $\lambda$, there exist two values of $\phi_{\pi}$, such that the REE is unique: one satisfies the Taylor principle, while the other does not. On the other hand, we can define threshold values for the share of non-Ricardian agents, such that: if $\lambda\left\langle\bar{\lambda}^{a, C R}\right.$, then $\phi_{\pi}>1$ is a sufficient (but not necessary) condition for the uniqueness of the REE; if $\lambda>\bar{\lambda}^{b, C R}$, then $\phi_{\pi}<1$ is a sufficient (but not necessary) condition for the uniqueness of the REE. These threshold values are given by the intersection between $\phi_{\pi}=1$, and $\bar{\phi}_{\pi}^{a, C R}$ and $\bar{\phi}_{\pi}^{b, C R}$, respectively (see Appendix A.2.1). ${ }^{7}$ Note that if $\beta \cong 1$, then $\bar{\phi}_{\pi}^{b, C R} \cong 0$, so that the standard Taylor principle holds for positive values of $\phi_{\pi}$, because the REE is always indeterminate for $0<\phi_{\pi}<1$, as in the standard case.

Wage stickiness shifts to the left both the $\bar{\phi}_{\pi}^{a, C R}$ and the $\bar{\phi}_{\pi}^{b, C R}$ curves in Figure 3, because $\frac{\partial \bar{\phi}_{\pi}^{a, C R}}{\partial \kappa_{w}}<0$ and $\frac{\partial \bar{\phi}_{\pi}^{b, C R}}{\partial \kappa_{w}}<0$. Hence, both the threshold values $\bar{\lambda}^{a, C R}$ and $\bar{\lambda}^{b, C R}$ increase with the degree of wage stickiness. Again as $\kappa_{w}$ tends to 0 (limiting case of fix wages), Proposition 2 collapses to the standard Taylor principle $\left(\phi_{\pi}>1\right)$, because $\phi_{\pi}^{a}$ and $\phi_{\pi}^{b}$ tend to $(-\infty)$. Both $\bar{\lambda}^{a, C R}$ and $\bar{\lambda}^{b, C R}$ are, instead, decreasing with the degree of price stickiness (i.e., increases with $\left.\kappa_{p}\right)$. In the limiting case of fully flexible prices $\left(\kappa_{p} \longrightarrow \infty\right)$, Proposition 2 defines the following condition for determinacy: either $\phi_{\pi}>1$ or $\phi_{\pi}<-1-\frac{2 \sigma(1+\beta)}{(\sigma+\phi) \kappa_{w}}$. Thus, as in the case of a forward rule, in an economy with flexible prices and sticky wages, the degree of LAMP has no effect on the shape of the determinacy regions, and the Taylor principle holds, at least for positive values of $\phi_{\pi}$.

From a numerical point of view, Figure 4 shows that the result in the Corollary is confirmed also in the case of contemporaneous rule: under sticky wages, the Taylor principle is a necessary and sufficient condition for the uniqueness of the REE, for all plausible values of $\lambda$ (abstracting from highly negative values of $\phi_{\pi}$ ). This is not the case instead when wages are flexible, since the $\bar{\phi}_{\pi}^{a, C R}$ curve shifts downward. Indeed, $\bar{\lambda}^{a, C R}=0.831$, in our standard calibration, while it lowers to 0.197 in the case of flexible wages. ${ }^{8}$

In a model that features both sticky wages and sticky prices, both Erceg et al. (2000) and Galí (2008) numerically study the properties of a monetary policy rule that targets both price and wage inflation, as $i_{t}=\phi_{\pi} \pi_{t}+\phi_{\pi^{w}} \pi_{t}^{w}$. Galí (2008) numerically shows that, for

\footnotetext{
${ }^{6}$ The Appendix shows that this Proposition assumes: $\frac{\sigma(1-\beta)\left[(\sigma+\phi) \kappa_{p} \kappa_{w}+4 \sigma(1+\beta)^{2}\right]}{(\sigma+\phi) \kappa_{p} \kappa_{w} \sigma[1+3 \beta]}<1$. This condition holds for value of $\beta$ sufficiently close to one and for our benchmark calibration.

${ }^{7}$ Depending on parameter values $\bar{\lambda}_{\pi}^{a, C R}$ can be larger or smaller than $\bar{\lambda}_{\pi}^{b, C R}$. In general, $\bar{\lambda}_{\pi}^{a, C R} \lessgtr \bar{\lambda}_{\pi}^{b, C R}$ iff $\frac{(1-\beta)(1+\beta)^{2}}{\beta} \lessgtr \frac{(\sigma+\phi) \kappa_{p} \kappa_{w}}{\sigma}$. Hence for values of $\beta$ sufficiently close to 1 , then $\bar{\lambda}_{\pi}^{a, C R}<\bar{\lambda}_{\pi}^{b, C R}$, as in Figure 3 .

${ }^{8}$ Moreover, similarly to the case of the forward-looking rule, Figure 4 reveals that the curve $\bar{\phi}_{\pi}^{a, C R}$ is flat at $\bar{\lambda}^{a, C R}$, given our standard calibration.
} 
$\phi_{\pi}, \phi_{\pi^{w}} \in(0, \infty)$, the condition $\phi_{\pi}+\phi_{\pi^{w}}>1$ is necessary and sufficient (see also Flaschel et al., 2008) for the uniqueness of the REE. Applying the proposed methodology, Proposition 3 shows analytically that such a condition is still crucial in a model with LAMP.

Proposition 3. Price Inflation and Wage Inflation Targeting Rule Let $i_{t}=\phi_{\pi} \pi_{t}+$ $\phi_{\pi^{w}} \pi_{t}^{w}$. A necessary condition for the REE to be determined is either $\left(\phi_{\pi}+\phi_{\pi^{w}}\right)>$ $\max \left\{1, \bar{\phi}_{\pi, \pi^{w}}\right\}$ or $\left(\phi_{\pi}+\phi_{\pi^{w}}\right)<\min \left\{1, \bar{\phi}_{\pi, \pi^{w}}\right\}$ where $\bar{\phi}_{\pi, \pi^{w}}=-1-\frac{2 \sigma(\beta+1)\left[2(\beta+1)+\left(\kappa_{p}+\kappa_{w}\right)-\frac{\lambda}{1-\lambda}(\sigma+\phi) \kappa_{w}+\frac{1}{\sigma}(\sigma+\phi) \kappa_{w} \phi_{\pi}\right]}{(\sigma+\phi)\left(1+\phi_{\pi}+\phi_{\pi} w\right) \kappa_{p} \kappa_{w}}$.

Proof. See Appendix A.2.2.

The conditions in Proposition 3 refers now to the sum $\left(\phi_{\pi}+\phi_{\pi^{w}}\right)$. The threshold value $\bar{\phi}_{\pi, \pi^{w}}$ is increasing in $\lambda$. Thus, it is possible to define a threshold for the share of non-Ricardian agents, such that if $\lambda$ is lower than this threshold, $\bar{\phi}_{\pi, \pi^{w}}<0$ so that $\left(\phi_{\pi}+\phi_{\pi^{w}}\right)>1$ is a necessary condition for the uniqueness of the REE for positive values of both $\phi_{\pi}$ and $\phi_{\pi^{w}}$. This is always true for either fix wages or flexible prices.

\section{Conclusions}

We show how to use Hurwitz polynomials to study the stability and uniqueness of REE in Dynamic General Equilibrium models. We apply this method to provide analytical conditions for stability and uniqueness of the REE in a fourth-order dynamic model characterized by staggered wage and price contracts and by an arbitrary degree of asset market participation. Our model nests two widely used framework for the analysis of monetary policy: (i) the LAMP model by Bilbiie (2008) and (ii) the sticky prices-sticky wages model by Erceg et al. (2000). Framework (i) features no wage stickiness and exhibits an inverted Taylor principle. Our results show that, once wage stickiness is considered, the determinacy properties of simple interest rules agree with the Taylor principle for values of the share of non-Ricardian agents consistent with the empirical estimates. This suggests that reappraisals of the conduct of monetary policy in specific past periods, such as that of the Great Inflation, based on the presence of non-Ricardian agents cannot neglect nominal wage stickiness, which is, in fact, an incontrovertible empirical fact.

\section{References}

Ascari, G., A. Colciago and L. Rossi (2015). Limited asset market participation and optimal monetary policy. mimeo.

Basu S. and J. Fernald (1997). Returns to scale in u.s. production: Estimates and implications. Journal of Political Economy 105(2), pages 249-83.

Bilbiie, F. O. (2008). Limited asset market participation, monetary policy and (inverted) aggregate demand logic. Journal of Economic Theory 140, 162-196.

Blanchard, O. J. and C. M. Kahn (1980). The solution of linear difference models under rational expectations. Econometrica 48, 1305-1311.

Colciago, A. (2011). Rule of thumb consumers meet sticky wages. Journal of Money Credit and Banking 43(2), 325-353. 
Erceg, C. J., D. W. Henderson, and A. T. Levin (2000). Optimal monetary policy with staggered wage and price contracts. Journal of Monetary Economics 46, 281-313.

Felippa, C. A. and K. C. Park (2004). Synthesis tools for structural dynamics and partitioned analysis of coupled systems. In A. Ibrahimbegovic and B. Brank (Eds.), Multi-Physics and Multi-Scale Computer Models in Nonlinear Analysis and Optimal Design of Engineering Structures under Extreme Conditions, Ljubliana, Slovenia, pp. 50-110. Proceedings NATO-ARW PST ARW980268. http://www.colorado.edu/engineering/CAS/Felippa.d/FelippaHome.d /Publications.d/Report.CU-CAS-05-05.pdf.

Flaschel, P., R. Franke, and C. R. Proano (2008). On equilibrium determinacy in New Keynesian models with staggered wage and price setting. The B.E. Journal of Macroeconomics, Topics 8(1), 1-10.

Galì, J. (2008). Monetary Policy, Inflation and the Business Cycle. Princeton University Press.

Galì, J., J. D. López-Salido, and J. Vallés (2004). Rule of thumb consumers and the design of interest rate rules. Journal of Money Credit and Banking 36(4), 739-764.

Galì, J., J. D. López-Salido, and J. Vallés (2007). Understanding the effects of government spending on consumption. Journal of the European Economic Association 5(1), 227 270 .

Levin, A. T., A. Onatski, J. C. Williams, and N. Williams (2005). Monetary policy under uncertainty in micro-founded macroeconometric models. NBER Macroeconomics Annual 20, 229-312.

Smets, F. and R. Wouters (2003). An estimated dynamic stochastic general equilibrium model of the euro area. Journal of the European Economic Association 1, 1124-1175. 


\section{A Technical Appendix}

\section{A.1 Forward Rule: Proof of Propositions 1}

We consider the following policy rule

$$
i_{t}=\phi_{\pi} \pi_{t+1}
$$

So the system is in matrix formulation (where $\left.\frac{\lambda}{1-\lambda}=\chi\right)$ :

$$
\left[\begin{array}{c}
\pi_{t+1}^{w} \\
\pi_{t+1} \\
x_{t+1} \\
\tilde{\omega}_{t+1}
\end{array}\right]=\underbrace{\left[\begin{array}{cccc}
\frac{1}{\beta} & 0 & -\frac{1}{\beta} \kappa_{w}(\sigma+\phi) & -\frac{1}{\beta} \kappa_{w} \\
0 & \frac{1}{\beta} & 0 & -\frac{1}{\beta} \kappa_{p} \\
\frac{1}{\beta} \chi & \frac{1}{\sigma} \frac{1}{\beta}\left(\phi_{\pi}-1\right)-\chi \frac{1}{\beta} & 1-\chi \frac{1}{\beta} \kappa_{w}(\sigma+\phi) & \chi \frac{1}{\beta}\left(\kappa_{w}+\kappa_{p}\right)-\frac{1}{\sigma} \frac{1}{\beta}\left(\phi_{\pi}-1\right) \kappa_{p} \\
\frac{1}{\beta} & -\frac{1}{\beta} & -\frac{1}{\beta} \kappa_{w}(\sigma+\phi) & 1+\frac{1}{\beta}\left(\kappa_{w}+\kappa_{p}\right)
\end{array}\right]}_{A}\left[\begin{array}{c}
\pi_{t}^{w} \\
\pi_{t} \\
x_{t} \\
\tilde{\omega}_{t}
\end{array}\right]
$$

The the coefficient of the characteristic polynomial are ${ }^{9}$

$a_{1}=-\operatorname{trace}(A)=-$ sum of the principal first-order minors of $A$

$a_{2}=$ sum of the principal second-order minors of $A$

$a_{3}=-$ sum of the principal third-order minors of $A$

$a_{4}=\operatorname{det}(A)$ (= principal of fourth-order).

The characteristic polynomial is then equal to

$$
\begin{aligned}
p(\gamma)= & \gamma^{4}+\left[\frac{1}{\beta}\left[-2-2 \beta-\left(\kappa_{w}+\kappa_{p}\right)+\chi \kappa_{w}(\sigma+\phi)\right]\right] \gamma^{3} \\
& +\left[\frac{1}{\beta}\left(\kappa_{p}+\kappa_{w}+\beta+1\right)-\frac{1}{\beta} \kappa_{w}(\sigma+\phi)\left(\chi\left(1+\frac{1}{\beta}\right)+\frac{1}{\sigma \beta} \kappa_{p}\left(\phi_{\pi}-1\right)\right)\right. \\
& \left.+\frac{1}{\beta^{2}}\left(1+3 \beta+\kappa_{w}+\kappa_{p}\right)\right] \gamma^{2} \\
& +\left[-\frac{1}{\beta^{2}}\left(2+2 \beta+\kappa_{w}+\kappa_{p}-\chi \kappa_{w}(\sigma+\phi)\right)\right] \gamma+\frac{1}{\beta^{2}}
\end{aligned}
$$

\footnotetext{
${ }^{9}$ Given an $n x n$ matrix $A$, kth order principal minors are the determinants of the $k x k$ submatrices along the diagonal obtained by deleting $n-k$ columns and the same $n-k$ rows from $A$.
} 
Applying the above transformation in (6) to get the Hurwitz polynomial, it yields

$$
\begin{aligned}
& \underbrace{\frac{-\frac{1}{\sigma} \kappa_{w} \kappa_{p}(\sigma+\phi)\left(\phi_{\pi}-1\right)}{D}}_{\tilde{a}_{3}} \\
& +s \underbrace{\frac{2(\beta-1)\left[-\kappa_{w}-\kappa_{p}+\chi \kappa_{w}(\sigma+\phi)\right]}{D}}_{\tilde{a}_{2}} \\
& +s^{2}[\underbrace{\frac{4 \beta^{2}+4-8 \beta+2(1+\beta)\left[-\kappa_{p}-\kappa_{w}+\kappa_{w}(\sigma+\phi) \chi\right]+\frac{2}{\sigma} \kappa_{w}(\sigma+\phi) \kappa_{p}\left(\phi_{\pi}-1\right)}{D}}_{\tilde{a}_{1}}]
\end{aligned}
$$

where

$$
D=4 \beta^{2}+4+8 \beta+2[\beta+1]\left(\kappa_{p}+\kappa_{w}\right)-\frac{1}{\sigma} \kappa_{w} \kappa_{p}(\sigma+\phi)\left(\phi_{\pi}-1\right)-2(1+\beta) \chi \kappa_{w}(\sigma+\phi) .
$$

Note there should be 3 positive roots and 1 negative root for the REE to be unique. It follows that a necessary condition must be that $\tilde{a}_{4}<0$. Proof strategy: we look at the signs of the coefficients $\tilde{a}_{i}$, and we exploit the Decartes' rule of sign.

Look separately at the case when $\phi_{\pi}>1$ and when $\phi_{\pi}<1$.

Case $\phi_{\pi}>1$.

$\tilde{a}_{4}$ ) In this case the numerator of $\tilde{a}_{4}$ (i.e., $\left.N_{\tilde{a}_{4}}\right)^{10}$ is negative, hence the denominator must be positive. For $D$ to be positive, the following restriction must hold:

$\phi_{\pi}<1+\frac{4 \sigma \beta^{2}+4 \sigma+8 \sigma \beta+2 \sigma(1+\beta)\left(\left[\kappa_{p}+\kappa_{w}-\chi \kappa_{w}(\sigma+\phi)\right]\right.}{\kappa_{w} \kappa_{p}(\sigma+\phi)}$.

$\left.\tilde{a}_{3}\right)$ Then, since $D>0$, there are two cases:

i) $N_{\tilde{a}_{3}}>0=>\tilde{a}_{3}>0$, that happens for low values of $\chi$, more precisely when $\chi<\frac{\kappa_{w}+\kappa_{p}}{\kappa_{w}(\sigma+\phi)}$.

Note that in this case $\frac{4 \sigma \beta^{2}+4 \sigma+8 \sigma \beta+2 \sigma(1+\beta)\left(\left[\kappa_{p}+\kappa_{w}-\chi \kappa_{w}(\sigma+\phi)\right]\right.}{\kappa_{w} \kappa_{p}(\sigma+\phi)}+1>1$ and so the set is non empty. Moreover $N_{\tilde{a}_{1}}=-8+8 \beta^{2}+2(1-\beta)\left[-\left(\kappa_{w}+\kappa_{p}\right)+\chi \kappa_{w}(\sigma+\phi)\right]<0=>\tilde{a}_{1}<0$.

Whatever the sign of $\tilde{a}_{2}$, the signs of the coefficients in (11) are: -,+,?,-,+. By Decartes' rule of sign, $P_{H}(s)$ then admits then 1 or 3 positive roots. However, $P_{H}(-s)=+,-$, ,,,++ , and hence there can be only one negative root. It follows that under the above conditions

$$
\begin{gathered}
\frac{2 \sigma(1+\beta)\left[2(1+\beta)+\kappa_{p}+\kappa_{w}-\chi \kappa_{w}(\sigma+\phi)\right]}{\kappa_{w} \kappa_{p}(\sigma+\phi)}+1>\phi_{\pi}>1 \\
\chi<\frac{\kappa_{w}+\kappa_{p}}{\kappa_{w}(\sigma+\phi)}
\end{gathered}
$$

the REE is determinate.

ii) $N_{\tilde{a}_{3}}<0=>\tilde{a}_{3}<0$, that happens for high values of $\chi$, more precisely when $\chi>\frac{\kappa_{w}+\kappa_{p}}{\kappa_{w}(\sigma+\phi)}$. In this case, however, the set $\frac{2 \sigma(1+\beta)\left[2(1+\beta)+\kappa_{p}+\kappa_{w}-\chi \kappa_{w}(\sigma+\phi)\right]}{\kappa_{w} \kappa_{p}(\sigma+\phi)}+1>\phi_{\pi}>1$ is non

\footnotetext{
${ }^{10} N$ stands for numerator, $D$ for denominator and the pedix for the correspondent coefficient $\tilde{a}_{i}$.
} 
empty iff $\chi<\frac{\kappa_{p}+\kappa_{w}}{\kappa_{w}(\sigma+\phi)}+\frac{2(1+\beta)}{\kappa_{w}(\sigma+\phi)}$. Hence now we are looking at values of $\chi$ such that

$$
\frac{\kappa_{p}+\kappa_{w}}{\kappa_{w}(\sigma+\phi)}+\frac{2(1+\beta)}{\kappa_{w}(\sigma+\phi)}>\chi>\frac{\kappa_{w}+\kappa_{p}}{\kappa_{w}(\sigma+\phi)}
$$

Since the first two coefficients $\left(\tilde{a}_{4}, \tilde{a}_{3}\right)$ are negative and the last is positive, it must be that $\tilde{a}_{2}>0$ and $\tilde{a}_{1}<0$ to have three signs inversions. This is always true if $\phi_{\pi}>1$ and (12) hold. It follows that under the above conditions

$$
\begin{gathered}
\frac{2 \sigma(1+\beta)\left[2(1+\beta)+\kappa_{p}+\kappa_{w}-\chi \kappa_{w}(\sigma+\phi)\right]}{\kappa_{w} \kappa_{p}(\sigma+\phi)}+1>\phi_{\pi}>1 \\
\frac{\kappa_{p}+\kappa_{w}}{\kappa_{w}(\sigma+\phi)}+\frac{2(1+\beta)}{\kappa_{w}(\sigma+\phi)}>\chi>\frac{\kappa_{w}+\kappa_{p}}{\kappa_{w}(\sigma+\phi)}
\end{gathered}
$$

the REE is determinate.

Putting together i) and ii), the equilibrium is determinate iff

$$
\frac{2 \sigma(1+\beta)\left[2(1+\beta)+\kappa_{p}+\kappa_{w}-\chi \kappa_{w}(\sigma+\phi)\right]}{\kappa_{w} \kappa_{p}(\sigma+\phi)}+1>\phi_{\pi}>1
$$

and

$$
\frac{\kappa_{p}+\kappa_{w}}{\kappa_{w}(\sigma+\phi)}+\frac{2(1+\beta)}{\kappa_{w}(\sigma+\phi)}>\chi>\frac{\kappa_{w}+\kappa_{p}}{\kappa_{w}(\sigma+\phi)}
$$

Case $\phi_{\pi}<1$.

$\left.\tilde{a}_{4}\right) N_{\tilde{a}_{4}}>0$, hence it must be that $D<0$. For $D$ to be negative, the following restriction must hold: $\frac{2 \sigma(1+\beta)\left[2(1+\beta)+\kappa_{p}+\kappa_{w}-\chi \kappa_{w}(\sigma+\phi)\right]}{\kappa_{w} \kappa_{p}(\sigma+\phi)}+1<\phi_{\pi}$. In this case, however, the set

$$
1>\phi_{\pi}>1+\frac{2 \sigma(1+\beta)\left[2(1+\beta)+\kappa_{p}+\kappa_{w}-\chi \kappa_{w}(\sigma+\phi)\right]}{\kappa_{w} \kappa_{p}(\sigma+\phi)}
$$

is non empty iff:

$$
\chi>\frac{\kappa_{p}+\kappa_{w}}{\kappa_{w}(\sigma+\phi)}+\frac{2(1+\beta)}{\kappa_{w}(\sigma+\phi)} .
$$

$\tilde{a}_{3}$ ) Given (16), $\Longrightarrow N_{\tilde{a}_{3}}<0=>\tilde{a}_{3}>0$, since $D<0$. In this case, since the first two coefficients: $\tilde{a}_{4}<0, \tilde{a}_{3}>0$, and the last is positive, the only way to have three signs inversions is that at least one between $\tilde{a}_{2}$ and $\tilde{a}_{1}$ is negative (in other words they cannot be both positive).

Condition for $\tilde{a}_{2}<0=>N_{\tilde{a}_{2}}>0=>$

$$
\phi_{\pi}>1-\frac{\sigma(1+\beta)\left[\kappa_{w}(\sigma+\phi) \chi-\kappa_{p}-\kappa_{w}\right]}{\kappa_{w}(\sigma+\phi) \kappa_{p}}-\frac{2 \sigma(1-\beta)^{2}}{\kappa_{w}(\sigma+\phi) \kappa_{p}}
$$

which, if (15) holds, is satisfied iff:

$$
\chi<\frac{\kappa_{p}+\kappa_{w}}{\kappa_{w}(\sigma+\phi)}+\frac{4(1+\beta)}{\kappa_{w}(\sigma+\phi)}+\frac{2(1-\beta)^{2}}{\kappa_{w}(\sigma+\phi)(1+\beta)} .
$$

In other words, (17) guarantees that

$$
\begin{gathered}
1+\frac{2 \sigma(1+\beta)\left[2(1+\beta)+\kappa_{p}+\kappa_{w}-\chi \kappa_{w}(\sigma+\phi)\right]}{\kappa_{w} \kappa_{p}(\sigma+\phi)}>1-\frac{\sigma(1+\beta)\left[\kappa_{w}(\sigma+\phi) \chi-\kappa_{p}-\kappa_{w}\right]}{\kappa_{w}(\sigma+\phi) \kappa_{p}}-\frac{2 \sigma(1-\beta)^{2}}{\kappa_{w}(\sigma+\phi) \kappa_{p}} . \\
\text { Condition for } \tilde{a}_{1}<0=>N_{\tilde{a}_{1}}>0=> \\
\chi>\frac{4(1+\beta)+\kappa_{w}+\kappa_{p}}{\kappa_{w}(\sigma+\phi)} .
\end{gathered}
$$


Note that: if (16) holds, at least one between (17) and/or (18) is satisfied, since $\frac{\kappa_{p}+\kappa_{w}}{\kappa_{w}(\sigma+\phi)}+$ $\frac{2(1+\beta)}{\kappa_{w}(\sigma+\phi)}<\frac{4(1+\beta)+\kappa_{w}+\kappa_{p}}{\kappa_{w}(\sigma+\phi)}<\frac{\kappa_{p}+\kappa_{w}}{\kappa_{w}(\sigma+\phi)}+\frac{4(1+\beta)}{\kappa_{w}(\sigma+\phi)}+\frac{2(1-\beta)^{2}}{\kappa_{w}(\sigma+\phi)(1+\beta)}$. Hence (16) guarantees that at least one between $\tilde{a}_{2}$ and $\tilde{a}_{1}$ is negative. Decartes' rule of signs then implies 3 positive roots.

To conclude, in the case $\phi_{\pi}<1$, the equilibrium is determinate iff

$$
1>\phi_{\pi}>1+\frac{2 \sigma(1+\beta)\left[2(1+\beta)+\kappa_{p}+\kappa_{w}-\chi \kappa_{w}(\sigma+\phi)\right]}{\kappa_{w} \kappa_{p}(\sigma+\phi)}
$$

and

$$
\chi>\frac{\kappa_{p}+\kappa_{w}}{\kappa_{w}(\sigma+\phi)}+\frac{2(1+\beta)}{\kappa_{w}(\sigma+\phi)} .
$$

Putting together the two cases $\phi_{\pi}>1$ and $\phi_{\pi}<1$., it yields Proposition 1. QED

\section{A.2 Contemporaneous Rule}

We consider the following policy rule:

$$
i_{t}=\phi_{\pi} \pi_{t}+\phi_{\pi^{w}} \pi_{t}^{w}
$$

The corresponding matrix formulation of our dynamic system is:

$$
\left[\begin{array}{c}
\pi_{t+1}^{w} \\
\pi_{t+1} \\
x_{t+1} \\
\tilde{\omega}_{t+1}
\end{array}\right]=\left[\begin{array}{cccc}
\frac{1}{\beta} & 0 & -\frac{1}{\beta} \kappa_{w}(\sigma+\phi) & +\frac{1}{\beta} \kappa_{w} \\
0 & \frac{1}{\beta} & 0 & -\frac{1}{\beta} \kappa_{p} \\
\frac{1}{\sigma} \phi_{\pi^{w}}+\frac{1}{\beta} \chi & \frac{1}{\sigma} \phi_{\pi}-\frac{1}{\sigma} \frac{1}{\beta}-\chi \frac{1}{\beta} & 1-\chi \frac{1}{\beta} \kappa_{w}(\sigma+\phi) & \chi \frac{1}{\beta}\left(\kappa_{w}+\kappa_{p}\right)+\frac{1}{\sigma} \frac{1}{\beta} \kappa_{p} \\
\frac{1}{\beta} & -\frac{1}{\beta} & -\frac{1}{\beta} \kappa_{w}(\sigma+\phi) & 1+\frac{1}{\beta}\left(\kappa_{w}+\kappa_{p}\right)
\end{array}\right]\left[\begin{array}{c}
\pi_{t}^{w} \\
\pi_{t} \\
x_{t} \\
\tilde{\omega}_{t}
\end{array}\right] .
$$

The coefficients of the characteristic polynomial are:

$$
\begin{gathered}
a_{1}=-\operatorname{trace}\left(J_{0}\right)=-\frac{1}{\beta}\left[2(1+\beta)+\left(\kappa_{p}+\kappa_{w}\right)-\chi \kappa_{w}(\sigma+\phi)\right] \\
a_{2}=1+\frac{4}{\beta}+\frac{1}{\beta^{2}}+\frac{1}{\beta}\left(1+\frac{1}{\beta}\right)\left(\kappa_{p}+\kappa_{w}\right)-\frac{1}{\beta}\left(1+\frac{1}{\beta}\right) \chi \kappa_{w}(\sigma+\phi)+\frac{1}{\sigma \beta}\left(\phi_{\pi^{w}}+\frac{1}{\beta} \kappa_{p}\right)(\phi+\sigma) \kappa_{w} \\
a_{3}=-\frac{2}{\beta}\left(\frac{1}{\beta}+1\right)-\frac{1}{\beta^{2}}\left(\kappa_{p}+\kappa_{w}\right)+\left(\frac{\chi}{\beta^{2}}-\frac{1}{\sigma \beta^{2}}(\sigma+\phi) \kappa_{p} \kappa_{w}\left(\phi_{\pi}+\phi_{\pi^{w}}\right)-\frac{1}{\sigma \beta}\left(1+\frac{1}{\beta}\right) \phi_{\pi^{w}}\right)(\phi+\sigma) \kappa_{w} \\
a_{4}=\frac{1}{\beta^{2}}\left(1+\frac{1}{\sigma}(\sigma+\phi) \kappa_{w} \phi_{\pi^{w}}\right) .
\end{gathered}
$$


Repeating the steps above in (6), the Hurwitz polynomial is given by:

$$
\begin{aligned}
& \underbrace{\frac{1}{\sigma}\left(\begin{array}{l}
\left.1-\left(\phi_{\pi}+\phi_{\pi^{w}}\right)\right)(\sigma+\phi) \kappa_{p} \kappa_{w} \\
D
\end{array}\right.}_{\tilde{a}_{4}} \\
& +s \underbrace{\frac{2(1-\beta)\left[\kappa_{p}+\kappa_{w}-\kappa_{w}\left(\phi_{\pi^{w}}+(\sigma+\phi) \chi\right)\right]+\frac{2}{\sigma} \kappa_{w}\left[(\sigma+\phi)\left(\phi_{\pi}+\phi_{\pi^{w}}\right) \kappa_{p}+(\beta-1) \phi \phi_{\pi^{w}}\right]}{D}}_{\tilde{a}_{3}} \\
& +s^{2} \underbrace{\frac{4(1-\beta)^{2}-2(1+\beta)\left[\kappa_{p}+\kappa_{w}-\chi \kappa_{w}(\sigma+\phi)\right]-\frac{2}{\sigma} \kappa_{w}(\sigma+\phi)\left[\kappa_{p}-(\beta-3) \phi_{\pi^{w}}\right]}{D}}_{\tilde{a}_{2}} \\
& +s^{3} \underbrace{8\left(\beta^{2}-1\right)+2(\beta-1)\left[\kappa_{p}+\kappa_{w}-(\sigma+\phi) \chi \kappa_{w}\right]-\frac{2}{\sigma}(\phi+\sigma) \kappa_{w}\left[(\beta+3) \phi_{\pi^{w}}-\kappa_{p}\left(\phi_{\pi}+\phi_{\pi^{w}}\right)\right]}_{\tilde{a}_{1}}
\end{aligned}
$$

where

$$
\begin{aligned}
D= & 2(1+\beta)\left[2(1+\beta)+\left(\kappa_{p}+\kappa_{w}\right)-(\sigma+\phi) \chi \kappa_{w}\right] \\
& +\frac{2}{\sigma}(\beta+1)(\sigma+\phi) \kappa_{w} \phi_{\pi^{w}}+\frac{1}{\sigma}(\sigma+\phi)\left(1+\phi_{\pi}+\phi_{\pi^{w}}\right) \kappa_{p} \kappa_{w}
\end{aligned}
$$

Note there should be 3 positive roots and 1 negative root for the REE to be unique. It follows that a necessary condition must be that $\tilde{a}_{4}<0$. As for the proof above in A.1, we look at the signs of the coefficients $\tilde{a}_{i}$, and we exploit the Decartes' rule of sign.

\section{A.2.1 Proof of Propositions 2: Case $\phi_{\pi^{w}}=0$}

If $\phi_{\pi^{w}}=0$, the Hurwitz polynomial is:

$$
\begin{aligned}
& \underbrace{\frac{\frac{1}{\sigma}\left(1-\phi_{\pi}\right)(\sigma+\phi) \kappa_{p} \kappa_{w}}{d e n}}_{\tilde{a}_{4}}+ \\
& \underbrace{s(1-\beta)\left[\left(\kappa_{p}+\kappa_{w}\right)-(\sigma+\phi) \chi \kappa_{w}\right]+\frac{2}{\sigma}(\sigma+\phi) \phi_{\pi} \kappa_{p} \kappa_{w}}_{\tilde{a}_{3}}+ \\
& s^{2} \underbrace{\frac{4(1-\beta)^{2}-2(1+\beta)\left[\left(\kappa_{p}+\kappa_{w}\right)-\chi \kappa_{w}(\sigma+\phi)\right]-\frac{2}{\sigma}(\sigma+\phi) \kappa_{p} \kappa_{w}}{d e n}}_{\tilde{a}_{2}}+ \\
& s^{3} \underbrace{\frac{8 \beta^{2}-8+2(\beta-1)\left[\left(\kappa_{p}+\kappa_{w}\right)-(\sigma+\phi) \chi \kappa_{w}\right]-\frac{2}{\sigma} \kappa_{p} \kappa_{w}(\phi+\sigma) \phi_{\pi}}{\operatorname{den}}}_{\tilde{a}_{1}} \\
& +s^{4}
\end{aligned}
$$

where

$$
\operatorname{den}=2(1+\beta)\left[2(1+\beta)+\left(\kappa_{p}+\kappa_{w}\right)-(\sigma+\phi) \chi \kappa_{w}\right]+\frac{1}{\sigma}(\sigma+\phi)\left(1+\phi_{\pi}\right) \kappa_{p} \kappa_{w}
$$


Look separately at the case when $\phi_{\pi}>1$ and when $\phi_{\pi}<1$.

Case $\phi_{\pi}>1$.

$\tilde{a}_{4}$ ) In this case $N_{\tilde{a}_{4}}<0$, hence $D$ must be positive. For $D$ to be positive, the following restriction must hold:

$$
\phi_{\pi}>-1-\frac{2 \sigma(1+\beta)\left[2(1+\beta)+\left(\kappa_{p}+\kappa_{w}\right)-(\sigma+\phi) \chi \kappa_{w}\right]}{(\sigma+\phi) \kappa_{p} \kappa_{w}}=\bar{\phi}_{\pi}^{a, C R} .
$$

$\left.\tilde{a}_{3}\right)$ Then, since $D>0$, there are two cases:

i) $N_{\tilde{a}_{3}}>0=>\tilde{a}_{3}>0$, that happens for:

$$
\phi_{\pi}>\frac{\sigma(1-\beta)\left[(\sigma+\phi) \chi \kappa_{w}-\left(\kappa_{p}+\kappa_{w}\right)\right]}{(\sigma+\phi) \kappa_{p} \kappa_{w}}=\bar{\phi}_{\pi}^{b, C R}
$$

Note that in this case $\tilde{a}_{1}<0$, since $\tilde{a}_{1}=-\tilde{a}_{3}-8\left(1-\beta^{2}\right)$. It follows that, whatever the sign of $\tilde{a}_{2}, P_{H}(s)$ exhibits three sign changes, while $P_{H}(-s)$ only one. So there will be 3 positive roots and 1 negative root. This proves that if is $\phi_{\pi}>\max \left\{1 ; \bar{\phi}_{\pi}^{a, C R} ; \bar{\phi}_{\pi}^{b, C R}\right\}$, the REE is determinate.

ii) $N_{\tilde{a}_{3}}<0=>\tilde{a}_{3}<0$, that happens for: $\phi_{\pi}<\frac{\sigma(1-\beta)\left[(\sigma+\phi) \chi \kappa_{w}-\left(\kappa_{p}+\kappa_{w}\right)\right]}{(\sigma+\phi) \kappa_{p} \kappa_{w}}=\bar{\phi}_{\pi}^{b, C R}$. Since anyway, it should be $\phi_{\pi}>1$ and $\phi_{\pi}>\bar{\phi}_{\pi}^{a, C R}$, the condition $\phi_{\pi}<\bar{\phi}_{\pi}^{b, C R}$ then requires $\bar{\phi}_{\pi}^{b, C R}>1$ and $\bar{\phi}_{\pi}^{b, C R}>\bar{\phi}_{\pi}^{a, C R}$ to have an interval where the 3 conditions are all jointly satisfied. This implies the following conditions:

$$
\chi>\frac{\kappa_{p}}{\sigma(1-\beta)}+\frac{\kappa_{p}+\kappa_{w}}{(\sigma+\phi) \kappa_{w}}
$$

and

$$
\chi<\frac{\sigma\left(\kappa_{p}+\kappa_{w}\right)[1+3 \beta]+(\sigma+\phi) \kappa_{p} \kappa_{w}+4 \sigma(1+\beta)^{2}}{\sigma(\sigma+\phi) \kappa_{w}[1+3 \beta]} .
$$

Under this conditions, since the first two coefficients $\left(\tilde{a}_{4}, \tilde{a}_{3}\right)$ are negative and the last is positive, then it must be that $\tilde{a}_{2}>0$ and $\tilde{a}_{1}<0$ to have three signs inversions.

Condition for $\tilde{a}_{2}>0=>N_{\tilde{a}_{2}}>0=>$

$$
\chi>\frac{\kappa_{p}}{\sigma(1+\beta)}-\frac{2(1-\beta)}{\kappa_{w}(\sigma+\phi)}+\frac{\kappa_{p}+\kappa_{w}}{\kappa_{w}(\sigma+\phi)} \text {. }
$$

Condition for $\tilde{a}_{1}<0=>N_{\tilde{a}_{1}}<0=>$

$\phi_{\pi}>\bar{\phi}_{\pi}^{b, C R}-\frac{4 \sigma\left(1-\beta^{2}\right)}{\kappa_{p} \kappa_{w}(\phi+\sigma)}$.

So determinacy can occur iff all the following conditions are jointly satisfied:

$$
\left\{\begin{array}{c}
\phi_{\pi}>-1-\frac{2 \sigma(1+\beta)\left[2(1+\beta)+\left(\kappa_{p}+\kappa_{w}\right)-(\sigma+\phi) \chi \kappa_{w}\right]}{(\sigma+\phi) \kappa_{p} \kappa_{w}}=\bar{\phi}_{\pi}^{a, C R} \\
\phi_{\pi}<\frac{\sigma(1-\beta)\left[(\sigma+\phi) \chi \kappa_{w}-\left(\kappa_{p}+\kappa_{w}\right)\right]}{(\sigma+\phi) \kappa_{p} \kappa_{w}}=\bar{\phi}_{\pi}^{b, C R} \\
\phi_{\pi}>1 \\
\phi_{\pi}>\bar{\phi}_{\pi}^{b, C R}-\frac{4 \sigma\left(1-\beta^{2}\right)}{\kappa_{p} \kappa_{w}(\phi+\sigma)} \\
\chi>\frac{\kappa_{p}}{\sigma(1+\beta)}-\frac{2(1-\beta)}{\kappa_{w}(\sigma+\phi)}+\frac{\kappa_{p}+\kappa_{w}}{\kappa_{w}(\sigma+\phi)} \\
\chi>\frac{\kappa_{p}}{\sigma(1-\beta)}+\frac{\kappa_{p}+\kappa_{w}}{(\sigma+\phi) \kappa_{w}} \\
\chi<\frac{\sigma\left(\kappa_{p}+\kappa_{w}\right)[1+3 \beta]+(\sigma+\phi) \kappa_{p} \kappa_{w}+4 \sigma(1+\beta)^{2}}{\sigma(\sigma+\phi) \kappa_{w}[1+3 \beta]}
\end{array}\right.
$$


It is easy to show that this case is extremely unlikely. First, since it should be $\bar{\phi}_{\pi}^{b, C R}$ $\frac{4 \sigma\left(1-\beta^{2}\right)}{\kappa_{p} \kappa_{w}(\phi+\sigma)}<\phi_{\pi}<\bar{\phi}_{\pi}^{b, C R}$, then if $\beta=1$ this case does not admit determinacy. Moreover, for $\beta \rightarrow 1$, also the set that define the conditions on $\chi$ becomes empty. Second, for our benchmark calibration for the conditions above that define the admissible values of $\chi$ imply: $\chi>\frac{\kappa_{p}}{\sigma(1+\beta)}-\frac{2(1-\beta)}{\kappa_{w}(\sigma+\phi)}+\frac{\kappa_{p}+\kappa_{w}}{\kappa_{w}(\sigma+\phi)}=0.42246 ; \chi>\frac{\kappa_{p}}{\sigma(1-\beta)}+\frac{\kappa_{p}+\kappa_{w}}{(\sigma+\phi) \kappa_{w}}=9.3275$; $\chi<\frac{\sigma\left(\kappa_{p}+\kappa_{w}\right)[1+3 \beta]+(\sigma+\phi) \kappa_{p} \kappa_{w}+4 \sigma(1+\beta)^{2}}{\sigma(\sigma+\phi) \kappa_{w}[1+3 \beta]}=4.8919$, that can not be jointly satisfied.

Finally, $\bar{\phi}_{\pi}^{a, C R}$ and $\bar{\phi}_{\pi}^{b, C R}$ are equal for a value of $\chi$ that implies $\bar{\phi}_{\pi}^{a, C R}=\bar{\phi}_{\pi}^{b, C R}$ $=\frac{\sigma(1-\beta)\left[(\sigma+\phi) \kappa_{p} \kappa_{w}+4 \sigma(1+\beta)^{2}\right]}{(\sigma+\phi) \kappa_{p} \kappa_{w} \sigma[1+3 \beta]}$. It is sufficient to assume that $\frac{\sigma(1-\beta)\left[(\sigma+\phi) \kappa_{p} \kappa_{w}+4 \sigma(1+\beta)^{2}\right]}{(\sigma+\phi) \kappa_{p} \kappa_{w} \sigma[1+3 \beta]}$ is less than one to get rid of this case. So in what follows we will assume this mild condition, that is very likely to be satisfied.

Case $\phi_{\pi}<1$.

$\left.\tilde{a}_{4}\right)$ In this case $N_{\tilde{a}_{4}}>0$, hence $D$ must be negative. Thus:

$$
\phi_{\pi}<-1-\frac{2 \sigma(1+\beta)\left[2(1+\beta)+\left(\kappa_{p}+\kappa_{w}\right)-(\sigma+\phi) \chi \kappa_{w}\right]}{(\sigma+\phi) \kappa_{p} \kappa_{w}}=\bar{\phi}_{\pi}^{a, C R}
$$

$\left.\tilde{a}_{3}\right)$ Then, since $D<0$, there are two cases:

i) $N_{\tilde{a}_{3}}<0=>\tilde{a}_{3}>0$, that happens for

$$
\phi_{\pi}<\frac{\sigma(1-\beta)\left[(\sigma+\phi) \chi \kappa_{w}-\left(\kappa_{p}+\kappa_{w}\right)\right]}{(\sigma+\phi) \kappa_{p} \kappa_{w}}=\bar{\phi}_{\pi}^{b, C R}
$$

Note that in this case $\tilde{a}_{1}<0$, since $\tilde{a}_{1}=-\tilde{a}_{3}-8\left(1-\beta^{2}\right)$. It follows that, whatever the sign of $\tilde{a}_{2}, P_{H}(s)$ exhibits three sign changes, while $P_{H}(-s)$ only one. So there will be 3 positive roots and 1 negative root. This proves that if is $\phi_{\pi}>\min \left\{1 ; \bar{\phi}_{\pi}^{a, C R} ; \bar{\phi}_{\pi}^{b, C R}\right\}$, the REE is determinate.

ii) $N_{\tilde{a}_{3}}>0=>\tilde{a}_{3}<0$, that happens for: $\phi_{\pi}>\frac{\sigma(1-\beta)\left[(\sigma+\phi) \chi \kappa_{w}-\left(\kappa_{p}+\kappa_{w}\right)\right]}{(\sigma+\phi) \kappa_{p} \kappa_{w}}=\bar{\phi}_{\pi}^{b, C R}$. Since anyway, it should be $\phi_{\pi}<1$ and $\phi_{\pi}<\bar{\phi}_{\pi}^{a, C R}$, the condition $\phi_{\pi}>\bar{\phi}_{\pi}^{b, C R}$ then requires $\bar{\phi}_{\pi}^{b, C R}<1$ and $\bar{\phi}_{\pi}^{b, C R}<\bar{\phi}_{\pi}^{a, C R}$ to have an interval where the 3 conditions are all jointly satisfied. This implies the following conditions:

$$
\chi>\frac{\kappa_{p}}{\sigma(1-\beta)}+\frac{\kappa_{p}+\kappa_{w}}{(\sigma+\phi) \kappa_{w}}
$$

and

$$
\chi<\frac{\sigma\left(\kappa_{p}+\kappa_{w}\right)[1+3 \beta]+(\sigma+\phi) \kappa_{p} \kappa_{w}+4 \sigma(1+\beta)^{2}}{\sigma(\sigma+\phi) \kappa_{w}[1+3 \beta]} .
$$

Under this conditions, since the first two coefficients $\left(\tilde{a}_{4}, \tilde{a}_{3}\right)$ are negative and the last is positive, then it must be that $\tilde{a}_{2}>0$ and $\tilde{a}_{1}<0$ to have three signs inversions.

Condition for $\tilde{a}_{2}>0=>N_{\tilde{a}_{2}}<0=>$

$$
\begin{aligned}
& \chi<\frac{\kappa_{p}}{\sigma(1+\beta)}-\frac{2(1-\beta)}{\kappa_{w}(\sigma+\phi)}+\frac{\kappa_{p}+\kappa_{w}}{\kappa_{w}(\sigma+\phi)} . \\
& \text { Condition for } \tilde{a}_{1}<0=>N_{\tilde{a}_{1}}>0=> \\
& \phi_{\pi}<\bar{\phi}_{\pi}^{b, C R}-\frac{4 \sigma\left(1-\beta^{2}\right)}{\kappa_{p} \kappa_{w}(\phi+\sigma)} .
\end{aligned}
$$

This latter condition, however, contradicts the condition above that yields $\tilde{a}_{3}<0$, that is: $\phi_{\pi}>\bar{\phi}_{\pi}^{b, C R}$. Hence this case does not admit determinacy of REE.

The two conditions that are necessary and sufficient for the determinacy of the equilibrium are therefore: $\phi_{\pi}>\max \left\{1 ; \bar{\phi}_{\pi}^{a, C R} ; \bar{\phi}_{\pi}^{b, C R}\right\}$ or $\phi_{\pi}<\min \left\{1 ; \bar{\phi}_{\pi}^{a, C R} ; \bar{\phi}_{\pi}^{b, C R}\right\}$. QED 


\section{A.2.2 Proof of Proposition 3: Case $\phi_{\pi^{w}} \neq 0$}

Let's now consider the general case, where the Hurwitz polynomial is (21).

A first important result is that $1=\phi_{\pi}+\phi_{\pi^{w}}$ identifies a zero root. This analytically suggests that the numerical result in Galí (2008) regarding the model of Erceg et al. (2000) still survives in a model with LAMP.

Here we are just looking for a necessary condition that involves $\phi_{\pi}+\phi_{\pi^{w}} \lessgtr 1$. We know that a necessary condition is $\tilde{a}_{4}<0$. This is satisfied iff:

1) either $N_{\tilde{a}_{4}}>0, D<0$

2) or $N_{\tilde{a}_{4}}<0, D>0$.

That implies:

1) either $\left(\phi_{\pi}+\phi_{\pi^{w}}\right)<\min \left\{1, \bar{\phi}_{\pi, \pi^{w}}\right\}$

$2)$ or $\left(\phi_{\pi}+\phi_{\pi^{w}}\right)>\max \left\{1, \bar{\phi}_{\pi, \pi^{w}}\right\}$.

QED. 


\section{B Figures}

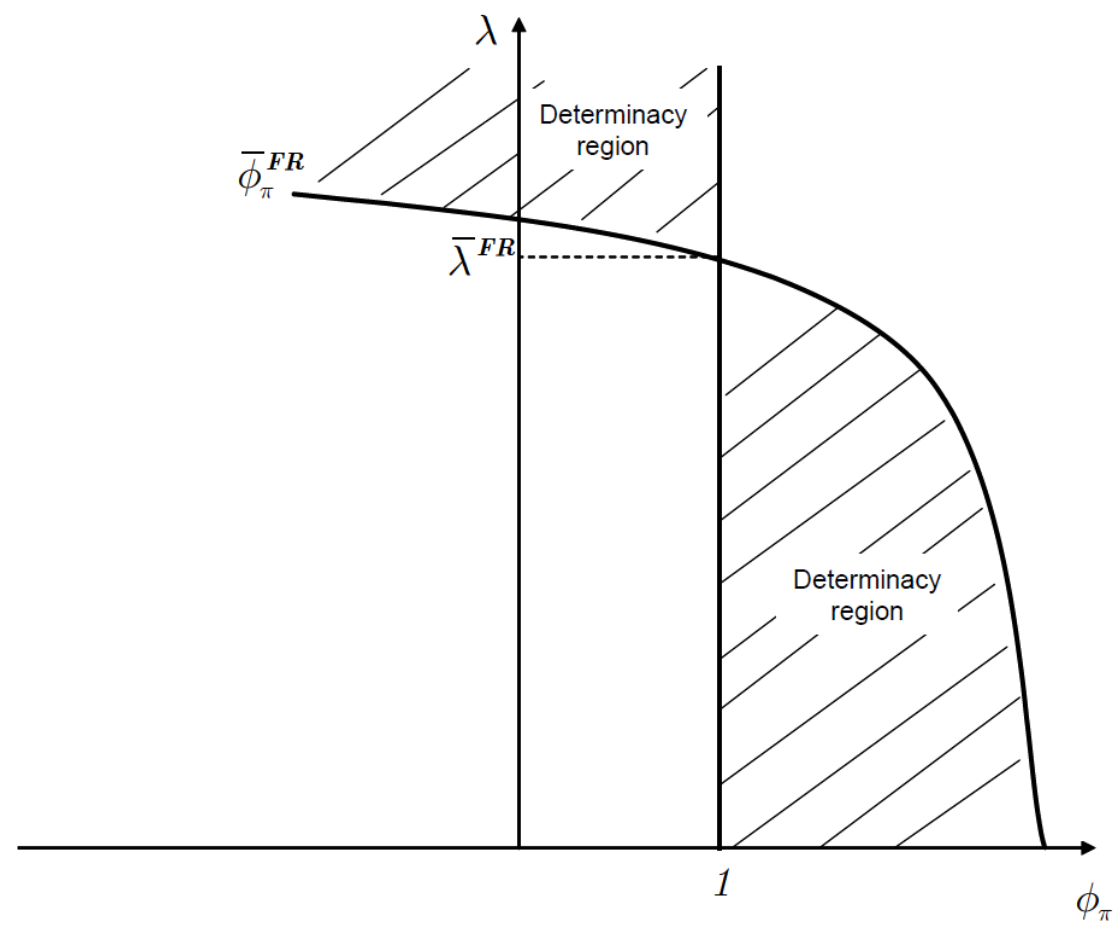

Figure 1. Determinacy and Indeterminacy regions when $i_{t}=\phi_{\pi} \pi_{t+1}$
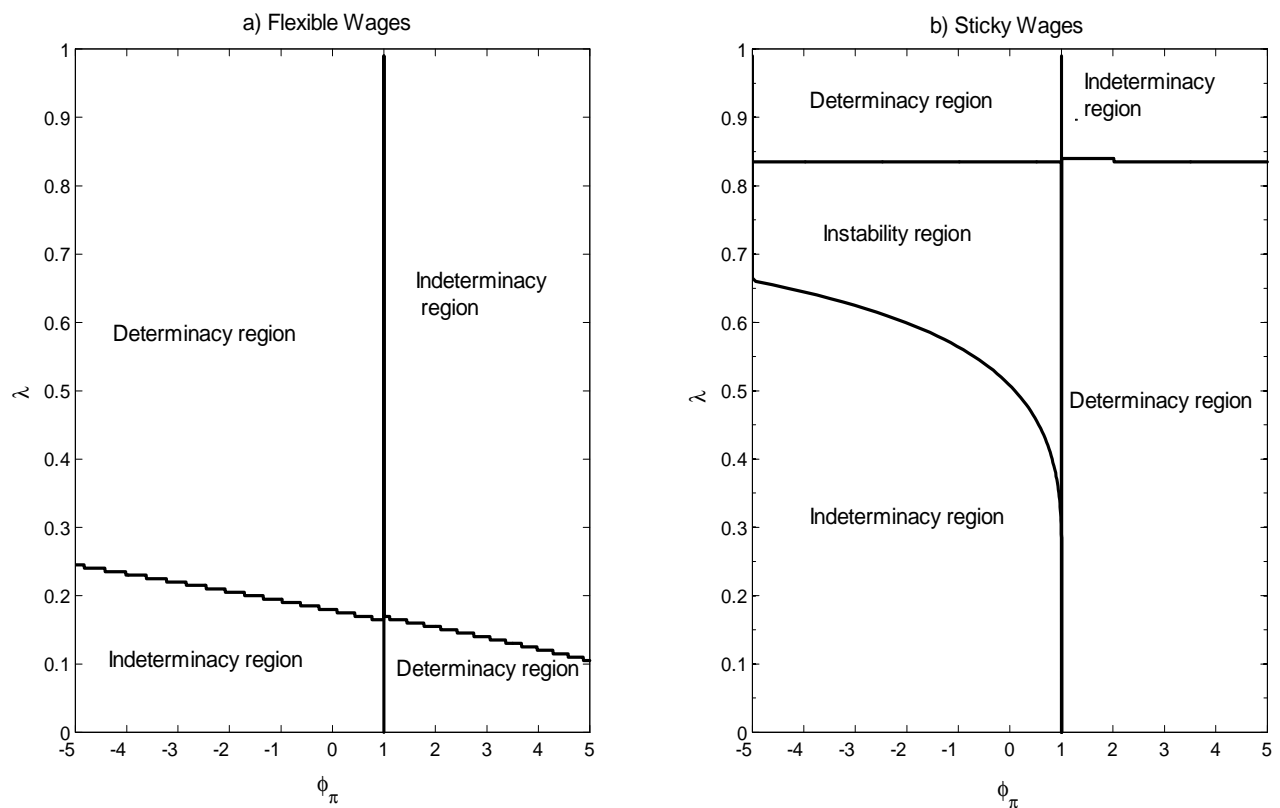

Figure 2. Determinacy and Indeterminacy regions under the rule: $i_{t}=\phi_{\pi} \pi_{t+1}$. Panel a): flexible wages, Panel b): sticky wages 


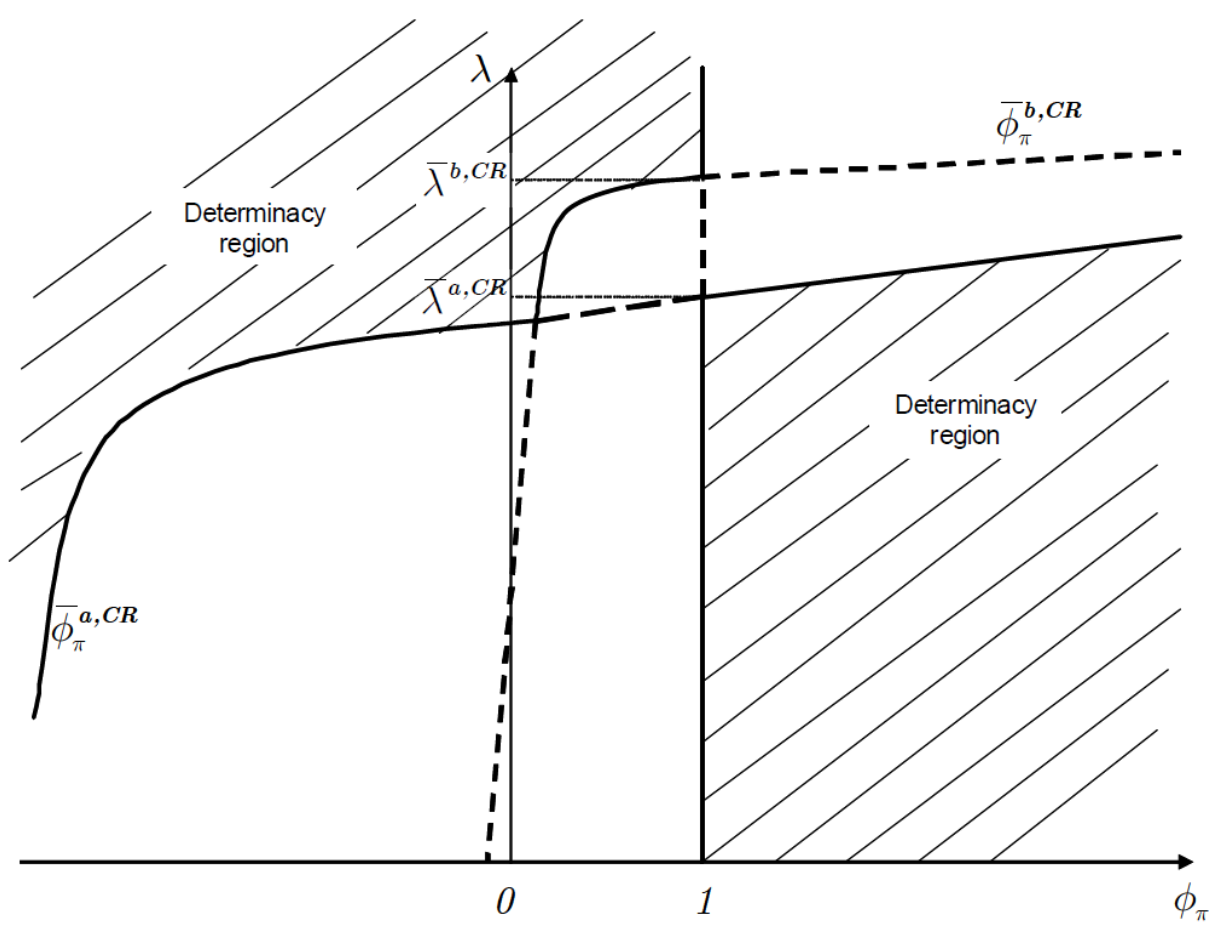

Figure 3. Determinacy and Indeterminacy regions when $i_{t}=\phi_{\pi} \pi_{t}$.
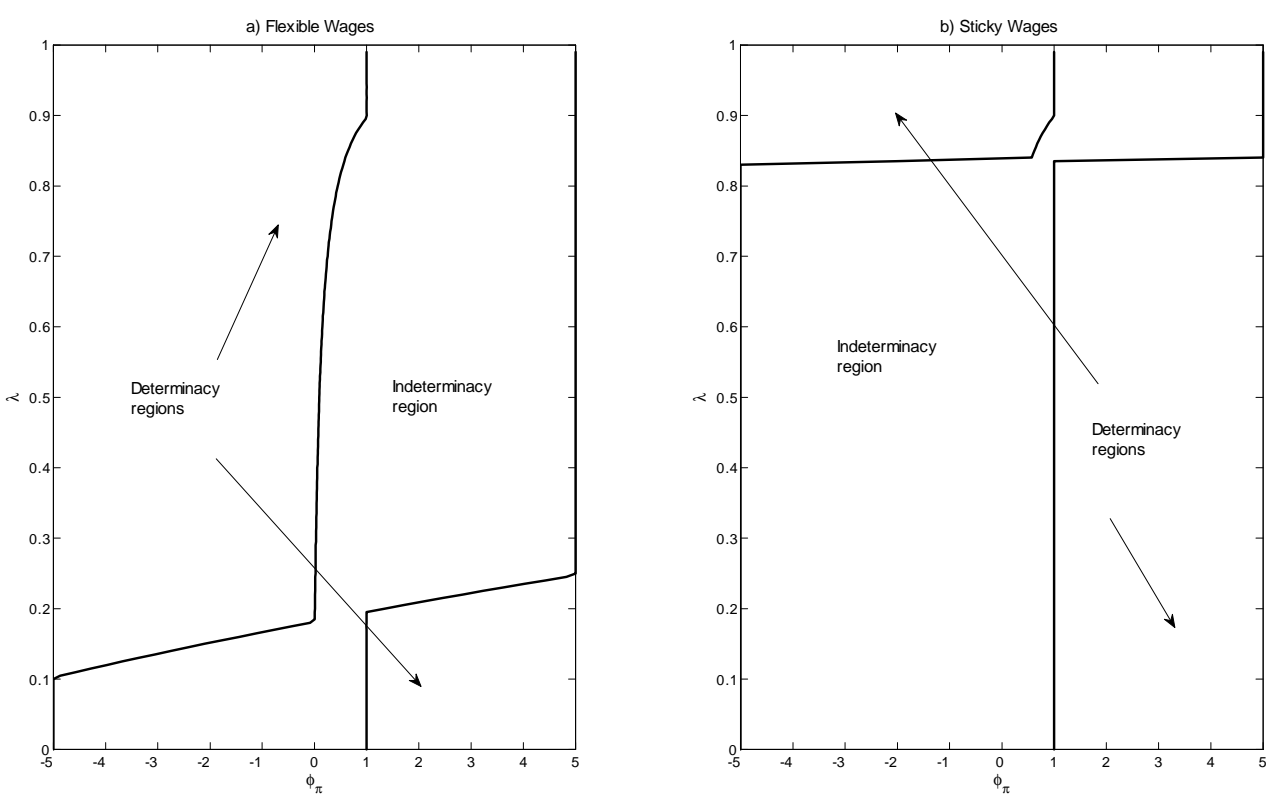

Figure 4. Determinacy and Indeterminacy regions under the rule: $i_{t}=\phi_{\pi} \pi_{t}$. Panel a): flexible wages, Panel b): sticky wages. 